Georgian Mathematical Journal

Volume 12 (2005), Number 4, 619-635

\title{
PROBLEMS OF STATICS OF TWO-COMPONENT ELASTIC MIXTURES
}

\author{
LEVAN GIORGASHVILI AND KETEVAN SKHVITARIDZE
}

\begin{abstract}
A general representation of solutions by six harmonic functions is obtained for a system of homogeneous equations of statics of two-component mixtures. The problems are investigated when the normal components of partial displacement vectors and the tangent components of partial rotation vectors are given on the boundary. Uniqueness theorems are proved. Solutions are obtained in terms of absolutely and uniformly convergent series.
\end{abstract}

2000 Mathematics Subject Classification. 35J55, 74H420, 74H25.

Key words and phrases. Theory of elasticity, theory of mixtures, Green formulas, regular functions.

\section{INTRODUCTION}

The effective solution of various boundary value and contact problems of the elasticity theory (of classical and and generalized models) is very important from theoretical and practical standpoints. Unfortunately, exact solutions of these problems can be constructed explicitly only for a rather limited number of bodies of concrete geometrical form. Here an essential role is played by the fact that a general solution of the system of complex differential equations that correspond to a mechanical model can be represented by solutions of simpler differential equations (of Laplace and Helmholtz). In the classical elasticity theory, to this topic the well-known works are devoted by such authors as W. Kelvin, J. Hadamard, J. Boussinesq, M. Pankovich, E. Trefftz, M. Slobodyanski, $\mathrm{N}$. Muskhelishvili, where the displacement vector is represented by harmonic and biharmonic functions. Such a representation makes it possible to develop the Fourier method of the partitioning of variables (with respect to the definite system of coordinates) of the elasticity theory in the case of canonical media.

The present paper deals with the representation of solutions of a system of differential equations of statics for elastic mixtures, with the construction of explicit solutions of a nonclassical problem for a ball and a hollow sphere, and with mathematical investigation of these solutions.

\section{Basic Equations and Formulation of the Boundary Value PROBLEM}

In the three-dimensional linear theory of elastic two-component mixtures, a system of homogeneous differential equations of statics is written in the form 
$([4],[7],[10])$

$$
\begin{aligned}
& a_{1} \Delta u^{\prime}+b_{1} \operatorname{grad} \operatorname{div} u^{\prime}+c \Delta u^{\prime \prime}+d \operatorname{grad} \operatorname{div} u^{\prime \prime}=0, \\
& c \Delta u^{\prime}+d \operatorname{grad} \operatorname{div} u^{\prime}+a_{2} \Delta u^{\prime \prime}+b_{2} \operatorname{grad} \operatorname{div} u^{\prime \prime}=0,
\end{aligned}
$$

where $u^{\prime}=\left(u_{1}^{\prime}, u_{2}^{\prime}, u_{3}^{\prime}\right), u^{\prime \prime}=\left(u_{1}^{\prime \prime}, u_{2}^{\prime \prime}, u_{3}^{\prime \prime}\right)$ are partial displacement vectors,

$$
\begin{gathered}
a_{1}=\mu_{1}-\lambda_{5}, \quad b_{1}=\mu_{1}+\lambda_{5}+\lambda_{1}-\frac{\rho_{2}}{\rho} \alpha^{\prime}, \quad a_{2}=\mu_{2}-\lambda_{5}, \\
b_{2}=\mu_{2}+\lambda_{2}+\lambda_{5}+\frac{\rho_{1}}{\rho} \alpha^{\prime}, \quad c=\mu_{3}+\lambda_{5}, \quad \alpha^{\prime}=\lambda_{3}-\lambda_{4}, \\
d=\mu_{3}+\lambda_{3}-\lambda_{5}-\frac{\rho_{1}}{\rho} \alpha^{\prime}, \quad \rho=\rho_{1}+\rho_{2},
\end{gathered}
$$

$\rho_{1}, \rho_{2}$ are partial densities of the mixture; $\lambda_{1}, \lambda_{2}, \ldots, \lambda_{5}, \mu_{1}, \mu_{2}, \mu_{3}$ are the elastic moduli characterizing the mechanical properties of the mixture, which satisfy the conditions [7]

$$
\begin{gathered}
\mu_{1}>0, \quad \mu_{1} \mu_{2}-\mu_{3}^{2}>0, \quad \lambda_{5}<0, \quad \lambda_{1}+\frac{2}{3} \mu_{1}-\frac{\rho_{2}}{\rho} \alpha^{\prime}>0 \\
\left(\lambda_{1}+\frac{2}{3} \mu_{1}-\frac{\rho_{2}}{\rho} \alpha^{\prime}\right)\left(\lambda_{2}+\frac{2}{3} \mu_{2}+\frac{\rho_{1}}{\rho} \alpha^{\prime}\right)>\left(\lambda_{3}+\frac{2}{3} \mu_{3}-\frac{\rho_{1}}{\rho} \alpha^{\prime}\right)^{2} .
\end{gathered}
$$

From these inequalities it follows that [7]

$$
d_{1}=\left(a_{1}+b_{1}\right)\left(a_{2}+b_{2}\right)-(c+d)^{2}>0, \quad d_{2}=a_{1} a_{2}-c^{2}>0 .
$$

Denote by $\Omega^{+}$the ball bounded by the spherical surface $\partial \Omega$ with center at the origin and radius $R$, i.e. $\Omega^{+}=\left\{x: x \in R^{3},|x|<R\right\}, \partial \Omega=\left\{x: x \in R^{3}\right.$, $|x|=R\}, \Omega^{-}=R^{3} \backslash \bar{\Omega}^{+}$.

Problem $(\mathbf{N})$. Find, in the domain $\Omega^{+}\left(\Omega^{-}\right)$, a regular solution $U(x)$ of system (1.1) that satisfies the following conditions on the boundary $\partial \Omega$ :

$$
\begin{array}{ll}
{\left[n(z) \cdot u^{\prime}(z)\right]^{ \pm}=f_{4}^{(1)}(z),} & {\left[n(z) \cdot u^{\prime \prime}(z)\right]^{ \pm}=f_{4}^{(2)}(z),} \\
{\left[n(z) \times \operatorname{rot} u^{\prime}(z)\right]^{ \pm}=f^{(1)}(z),} & {\left[n(z) \times \operatorname{rot} u^{\prime \prime}(z)\right]^{ \pm}=f^{(2)}(z),}
\end{array}
$$

where $U=\left(u^{\prime}, u^{\prime \prime}\right), f^{(j)}(z)=\left(f_{1}^{(j)}(z), f_{2}^{(j)}(z), f_{3}^{(j)}(z)\right), j=1,2, f_{k}^{(j)}(z), j=1,2$, $k=1,2,3,4$, are the functions given on $\partial \Omega, n(z)$ is the outward normal vector with respect to $\Omega^{+}$at a point $z \in \partial \Omega$.

Denote by $(N)^{+}$the internal problem, and by $(N)^{-}$the external one.

In the case of problem $(N)^{-}$, the vector $U(x)$ near the point at infinity should satisfy the following conditions:

$$
\begin{array}{ll}
u_{j}^{\prime}(x)=O\left(|x|^{-1}\right), & u_{j}^{\prime \prime}(x)=O\left(|x|^{-1}\right), \\
\frac{\partial u_{j}^{\prime}(x)}{\partial x_{k}}=o\left(|x|^{-1}\right), & \frac{\partial u_{j}^{\prime \prime}(x)}{\partial x_{k}}=o\left(|x|^{-1}\right), \quad j, k=1,2,3 .
\end{array}
$$

Note that estimates (1.5) follow from estimates (1.4) (see [1]).

Definition 1.1. The vector $U=\left(u^{\prime}, u^{\prime \prime}\right)$ defined in the domain $\Omega^{ \pm}$is called regular if $u_{k}^{\prime}, u_{k}^{\prime \prime} \in C^{2}\left(\Omega^{ \pm}\right) \cap C^{1}\left(\bar{\Omega}^{ \pm}\right), k=1,2,3$. 


\section{The Uniqueness TheOrem}

Theorem 2.1. If $\partial \Omega \in \Lambda_{1}(\alpha), 0<\alpha \leq 1$, then the homogeneous problem $(N)_{0}^{ \pm}$has only a trivial solution in the class of regular vectors.

Proof. Let us introduce the matrix differential operator $A(\partial x)$ :

$$
\begin{aligned}
& A(\partial x)=\left[\begin{array}{ccc}
A^{(1)}(\partial x) & \vdots & A^{(2)}(\partial x) \\
\ldots \ldots \ldots \ldots \ldots \ldots \ldots \ldots \\
A^{(3)}(\partial x) & \vdots & A^{(4)}(\partial x)
\end{array}\right]_{6 \times 6} \\
& A^{(i)}(\partial x)=\left[A_{k j}^{(i)}(\partial x)\right]_{3 \times 3}, \quad i=1,2,3,4, \\
& A_{k j}^{(1)}=a_{1} \delta_{k j} \Delta+b_{1} \frac{\partial^{2}}{\partial x_{k} \partial x_{j}}, \\
& A_{k j}^{(i)}=c \delta_{k j} \Delta+d \frac{\partial^{2}}{\partial x_{k} \partial x_{j}}, \quad i=2,3, \\
& A_{k j}^{(4)}=a_{2} \delta_{k j} \Delta+b_{2} \frac{\partial^{2}}{\partial x_{k} \partial x_{j}},
\end{aligned}
$$

where $\delta_{k j}$ is the Kronecker's symbol and $\Delta$ is the Laplace operator.

Using these notations, we rewrite system (1.1) as $A(\partial x) U(x)=0$.

Let us consider the scalar derivative

$$
\begin{aligned}
U \cdot A(\partial x) U= & \left(a_{1} u^{\prime}+c u^{\prime \prime}\right) \cdot \Delta u^{\prime}+\left(c u^{\prime}+a_{2} u^{\prime \prime}\right) \cdot \Delta u^{\prime \prime} \\
& +\left(b_{1} u^{\prime}+d u^{\prime \prime}\right) \cdot \operatorname{grad} \operatorname{div} u^{\prime}+\left(d u^{\prime}+b_{2} u^{\prime \prime}\right) \cdot \operatorname{grad} \operatorname{div} u^{\prime \prime} .
\end{aligned}
$$

Assume that $u=\left(u_{1}, u_{2}, u_{3}\right)$ and $v=\left(v_{1}, v_{2}, v_{3}\right)$ are three-component vectors. Then, after performing some transformations, we obtain

$$
\begin{aligned}
& u \cdot \Delta v=\operatorname{div}(u \operatorname{div} v)-\operatorname{div} u \operatorname{div} v+\operatorname{div}[u \times \operatorname{rot} v]-\operatorname{rot} u \cdot \operatorname{rot} v, \\
& u \cdot \operatorname{grad} \operatorname{div} v=\operatorname{div}(u \operatorname{div} v)-\operatorname{div} u \operatorname{div} v
\end{aligned}
$$

Substituting these equalities into (2.1), we have

$$
\begin{aligned}
U \cdot A(\partial x) U= & \operatorname{div}\left[\left(\left(a_{1}+b_{1}\right) u^{\prime}+(c+d) u^{\prime \prime}\right) \operatorname{div} u^{\prime}+\left((c+d) u^{\prime}\right.\right. \\
& \left.+\left(a_{2}+b_{2}\right) u^{\prime \prime}\right] \operatorname{div} u^{\prime \prime}+a_{1}\left(u^{\prime} \times \operatorname{rot} u^{\prime}\right)+c\left(u^{\prime} \times \operatorname{rot} u^{\prime \prime}\right) \\
& \left.+c\left(u^{\prime \prime} \times \operatorname{rot} u^{\prime}\right)+a_{2}\left(u^{\prime \prime} \times \operatorname{rot} u^{\prime \prime}\right)\right]-E(U, U),
\end{aligned}
$$

where

$$
\begin{aligned}
E(U, U)= & \frac{1}{a_{1}+b_{1}}\left[\left(\left(a_{1}+b_{1}\right) \operatorname{div} u^{\prime}+(c+d) \operatorname{div} u^{\prime \prime}\right)^{2}+d_{1}\left(\operatorname{div} u^{\prime \prime}\right)^{2}\right] \\
& +\frac{1}{a_{1}}\left[\left(a_{1} \operatorname{rot} u^{\prime}+c \operatorname{rot} u^{\prime \prime}\right)^{2}+d_{2}\left(\operatorname{rot} u^{\prime \prime}\right)^{2}\right] .
\end{aligned}
$$

Applying the Gauss-Ostrogradski theorem, from (2.2) we obtain

$$
\int_{\Omega^{+}} U \cdot A(\partial x) U d x=\int_{\partial \Omega}[U(z)]^{+} \cdot[P(\partial z, n) U(z)]^{+} d s-\int_{\Omega^{+}} E(U, U) d x,
$$


where

$$
\begin{aligned}
U \cdot P(\partial z, n) U= & \left(n \cdot u^{\prime}\right)\left[\left(a_{1}+b_{1}\right) \operatorname{div} u^{\prime}+(c+d) \operatorname{div} u^{\prime \prime}\right] \\
& +\left(n \cdot u^{\prime \prime}\right)\left[(c+d) \operatorname{div} u^{\prime}+\left(a_{2}+b_{2}\right) \operatorname{div} u^{\prime \prime}\right]- \\
& -\left(a_{1} u^{\prime}+c u^{\prime \prime}\right) \cdot\left[n \times \operatorname{rot} u^{\prime}\right]-\left(c u^{\prime}+a_{2} u^{\prime \prime}\right) \cdot\left[n \times \operatorname{rot} u^{\prime \prime}\right] .
\end{aligned}
$$

Here we have used the identity

$$
n \cdot[u \times \operatorname{rot} v]=-u \cdot[n \times \operatorname{rot} v] .
$$

Quite analogously, considering the domain $\Omega^{-}$and taking into account that the vector $U=\left(u^{\prime}, u^{\prime \prime}\right)$ satisfies condition (1.4)-(1.5), we obtain

$$
\int_{\Omega^{-}} U \cdot A(\partial x) U d x=-\int_{\partial \Omega}[U(z)]^{-} \cdot[P(\partial z, n) U(z)]^{-} d s-\int_{\Omega^{-}} E(U, U) d x .
$$

Let us consider the homogeneous problem $(N)_{0}^{ \pm}\left(f_{4}^{(j)}(z)=0, f^{(j)}(z)=0\right.$, $j=1,2)$. Applying the boundary conditions of problem $(N)_{0}^{ \pm}$to formula $(2.5)$, we obtain

$$
[U(z)]^{ \pm} \cdot[P(\partial z, n) U(z)]^{ \pm}=0, \quad z \in \partial \Omega .
$$

Using this equality in (2.4) and (2.6), we have

$$
\int_{\Omega^{ \pm}} U \cdot A(\partial x) U d x=-\int_{\Omega^{ \pm}} E(U, U) d x .
$$

Since (1.1) implies that $A(\partial x) U(x)=0, x \in \Omega^{ \pm}$, the latter equality yields

$$
\int_{\Omega^{ \pm}} E(U, U) d x=0
$$

Taking into account that $a_{1}>0, a_{1}+b_{1}>0, d_{1}>0, d_{2}>0$, from (2.3) it follows that $E(U, U) \geq 0$. By virtue of this fact, (2.7) implies

$$
E(U, U)=0, \quad x \in \Omega^{ \pm} \text {. }
$$

Hence, taking into account (2.3) we obtain

$\operatorname{div} u^{\prime}(x)=0, \quad \operatorname{div} u^{\prime \prime}(x)=0, \quad \operatorname{rot} u^{\prime}(x)=0, \quad \operatorname{rot} u^{\prime \prime}=0, \quad x \in \Omega^{ \pm}$.

A solution of this system has the form

$$
u^{\prime}(x)=\operatorname{grad} \Psi_{1}(x), \quad u^{\prime \prime}(x)=\operatorname{grad} \Psi_{2}(x), \quad x \in \Omega^{ \pm},
$$

where $\Psi_{j}(x), j=1,2$, is an arbitrary harmonic function.

Since $\left[n(z) \cdot u^{\prime}(z)\right]^{ \pm}=0,\left[n(z) \cdot u^{\prime \prime}(z)\right]^{ \pm}=0$, the harmonic functions $\Psi_{j}(x)$, $j=1,2$, satisfy, on the boundary $\partial \Omega$, the Neumann condition

$$
\left[\frac{\partial \Psi_{j}(x)}{\partial n(z)}\right]^{ \pm}=0, \quad z \in \partial \Omega
$$

As is known, the homogeneous Neumann problem has the solution $\Psi_{j}(x)=$ $C_{j}=$ const, $j=1,2, x \in \Omega^{ \pm}$. Substituting this value of $\Psi_{j}(x)$ into $(2.8)$, we obtain $u^{\prime}(x)=0, u^{\prime \prime}(x)=0, x \in \Omega^{ \pm}$. 
Thus the homogeneous problem $(N)_{0}^{ \pm}$has only a trivial solution. Hence it follows that problem $(N)^{ \pm}$admits no more than one regular solution.

\section{Representation of a Solution of System (1.1)}

Theorem 3.1. For the vector $U=\left(u^{\prime}, u^{\prime \prime}\right)$ to be a solution of the system of differential equations (1.1) in the domain $\Omega \subset R^{3}$, it is necessary and sufficient that it be represented in the form

$$
\begin{aligned}
u^{\prime}(x)= & \operatorname{grad} \Phi_{1}(x)+\operatorname{grad} r^{2}\left(r \frac{\partial}{\partial r}+1\right)\left[\alpha_{1} \Phi_{2}(x)+\beta_{1} \Phi_{3}(x)\right] \\
& +\operatorname{rot} \operatorname{rot}\left(x r^{2} \Phi_{2}(x)\right)+\operatorname{rot}\left(x \Phi_{5}(x)\right), \\
u^{\prime \prime}(x)= & \operatorname{grad} \Phi_{4}(x)+\operatorname{grad} r^{2}\left(r \frac{\partial}{\partial r}+1\right)\left[\beta_{2} \Phi_{2}(x)+\alpha_{2} \Phi_{3}(x)\right] \\
& +\operatorname{rot} \operatorname{rot}\left(x r^{2} \Phi_{3}(x)\right)+\operatorname{rot}\left(x \Phi_{6}(x)\right),
\end{aligned}
$$

where $\Phi_{j}(x), j=1,2, \ldots, 6$, are scalar harmonic functions, $x=\left(x_{1}, x_{2}, x_{3}\right)$, $r=|x|, r \frac{\partial}{\partial r}=x \cdot \operatorname{grad}, \alpha_{1}=\frac{1}{d_{1}}\left[c(c+d)-a_{1}\left(a_{2}+b_{2}\right)\right], \beta_{1}=\frac{1}{d_{1}}\left(a_{2} d-c b_{2}\right)$, $\beta_{2}=\frac{1}{d_{1}}\left(a_{1} d-c b_{1}\right), \alpha_{2}=\frac{1}{d_{1}}\left[c(c+d)-a_{2}\left(a_{1}+b_{1}\right)\right]$.

Proof. Applying the operation div to both equations of system (1.1), we obtain

$$
\begin{aligned}
& \left(a_{1}+b_{1}\right) \Delta \theta^{\prime}+(c+d) \Delta \theta^{\prime \prime}=0, \\
& (c+d) \Delta \theta^{\prime}+\left(a_{2}+b_{2}\right) \Delta \theta^{\prime \prime}=0,
\end{aligned}
$$

where we have introduced the notations

$$
\operatorname{div} u^{\prime}=\theta^{\prime}, \quad \operatorname{div} u^{\prime \prime}=\theta^{\prime \prime} .
$$

Since $d_{1}=\left(a_{1}+b_{1}\right)\left(a_{2}+b_{2}\right)-(c+d)^{2}>0$, from (3.2) we obtain $\Delta \theta^{\prime}=0$, $\Delta \theta^{\prime \prime}=0$.

Rewrite system (1.1) as

$$
\begin{aligned}
& a_{1} \Delta u^{\prime}+c \Delta u^{\prime \prime}=-b_{1} \operatorname{grad} \theta^{\prime}-d \operatorname{grad} \theta^{\prime \prime}, \\
& c \Delta u^{\prime}+a_{2} \Delta u^{\prime \prime}=-d \operatorname{grad} \theta^{\prime}-b_{2} \operatorname{grad} \theta^{\prime \prime} .
\end{aligned}
$$

Having solved this system, we obtain

$$
\begin{aligned}
& \Delta u^{\prime}=\frac{1}{d_{2}}\left[\left(c d-b_{1} a_{2}\right) \operatorname{grad} \theta^{\prime}+\left(c b_{2}-a_{2} d\right) \operatorname{grad} \theta^{\prime \prime}\right], \\
& \Delta u^{\prime \prime}=\frac{1}{d_{2}}\left[\left(c b_{1}-a_{1} d\right) \operatorname{grad} \theta^{\prime}+\left(c d-a_{1} b_{2}\right) \operatorname{grad} \theta^{\prime \prime}\right] .
\end{aligned}
$$

Hence we have

$$
\begin{aligned}
u^{\prime}(x) & =x\left(\sigma_{1} \theta^{\prime}+\sigma_{2} \theta^{\prime \prime}\right)+\Psi^{\prime}(x), \\
u^{\prime \prime}(x) & =x\left(\sigma_{3} \theta^{\prime}+\sigma_{4} \theta^{\prime \prime}\right)+\Psi^{\prime \prime}(x),
\end{aligned}
$$

where $\Psi^{\prime}(x), \Psi^{\prime \prime}(x)$ are arbitrary harmonic vectors,

$$
\sigma_{1}=\frac{1}{2 d_{2}}\left(c d-b_{1} a_{2}\right), \quad \sigma_{2}=\frac{1}{2 d_{2}}\left(c b_{2}-a_{2} d\right)
$$




$$
\sigma_{3}=\frac{1}{2 d_{2}}\left(c b_{1}-a_{1} d\right), \quad \sigma_{4}=\frac{1}{2 d_{2}}\left(c d-a_{1} b_{2}\right) .
$$

The substitution of the vectors $u^{\prime}(x)$ and $u^{\prime \prime}(x)$ from (3.4) into (3.3) gives

$$
\begin{gathered}
{\left[\sigma_{1}\left(r \frac{\partial}{\partial r}+3\right)-1\right] \theta^{\prime}+\sigma_{2}\left(r \frac{\partial}{\partial r}+3\right) \theta^{\prime \prime}=-\operatorname{div} \Psi^{\prime},} \\
\sigma_{3}\left(r \frac{\partial}{\partial r}+3\right) \theta^{\prime}+\left[\sigma_{4}\left(r \frac{\partial}{\partial r}+3\right)-1\right] \theta^{\prime \prime}=-\operatorname{div} \Psi^{\prime \prime} .
\end{gathered}
$$

The following statement is true [3].

Theorem 3.2. For the vector $\Psi(x)$ to be harmonic in the domain $\Omega \subset R^{3}$, it is necessary and sufficient that it be represented in the form

$$
\Psi(x)=\operatorname{grad} \Psi_{1}(x)+\operatorname{grad} r^{2} \Psi_{2}(x)-x\left(2 r \frac{\partial}{\partial r}+3\right) \Psi_{2}(x)+\operatorname{rot}\left(x \Psi_{3}(x)\right),
$$

where $\Psi_{j}(x), j=1,2,3$, are harmonic scalar functions, i.e., $\Delta \Psi_{j}(x)=0$, $j=1,2,3, x=\left(x_{1}, x_{2}, x_{3}\right), r \frac{\partial}{\partial r}=x \cdot \operatorname{grad}, r=|x|$.

Taking this theorem into account, we can write

$$
\begin{aligned}
& \Psi^{\prime}(x)=\operatorname{grad} \Phi_{1}(x)+\operatorname{grad} r^{2} \Psi_{2}^{\prime}(x)-x\left(2 r \frac{\partial}{\partial r}+3\right) \Psi_{2}^{\prime}(x)+\operatorname{rot}\left(x \Phi_{5}(x)\right), \\
& \Psi^{\prime \prime}(x)=\operatorname{grad} \Phi_{4}(x)+\operatorname{grad} r^{2} \Psi_{2}^{\prime \prime}(x)-x\left(2 r \frac{\partial}{\partial r}+3\right) \Psi_{2}^{\prime \prime}(x)+\operatorname{rot}\left(x \Phi_{6}(x)\right),
\end{aligned}
$$

where $\Delta \Phi_{j}(x)=0, \Delta \Psi_{2}^{\prime}(x)=0, \Delta \Psi_{2}^{\prime \prime}(x)=0, j=1,4,5,6$.

From (3.6) we have

$$
\begin{aligned}
& \operatorname{div} \Psi^{\prime}(x)=-\left(2 r \frac{\partial}{\partial r}+3\right)\left(r \frac{\partial}{\partial r}+1\right) \Psi_{2}^{\prime}(x), \\
& \operatorname{div} \Psi^{\prime \prime}(x)=-\left(2 r \frac{\partial}{\partial r}+3\right)\left(r \frac{\partial}{\partial r}+1\right) \Psi_{2}^{\prime \prime}(x) .
\end{aligned}
$$

Using these equalities in (3.5), we obtain

$$
\begin{gathered}
{\left[\sigma_{1}\left(r \frac{\partial}{\partial r}+3\right)-1\right] \theta^{\prime}+\sigma_{2}\left(r \frac{\partial}{\partial r}+3\right) \theta^{\prime \prime}=\left(2 r \frac{\partial}{\partial r}+3\right)\left(r \frac{\partial}{\partial r}+1\right) \Psi_{2}^{\prime},} \\
\sigma_{3}\left(r \frac{\partial}{\partial r}+3\right) \theta^{\prime}+\left[\sigma_{4}\left(r \frac{\partial}{\partial r}+3\right)-1\right] \theta^{\prime \prime}=\left(2 r \frac{\partial}{\partial r}+3\right)\left(r \frac{\partial}{\partial r}+1\right) \Psi_{2}^{\prime \prime} .
\end{gathered}
$$

Equalities (3.7) are fulfilled if the functions $\theta^{\prime}, \theta^{\prime \prime}, \Psi_{2}^{\prime}, \Psi_{2}^{\prime \prime}$ are chosen as follows:

$$
\begin{aligned}
\theta^{\prime}(x) & =\left(2 r \frac{\partial}{\partial r}+3\right)\left(r \frac{\partial}{\partial r}+1\right) \Phi_{2}^{\prime}(x) \\
\theta^{\prime \prime}(x) & =\left(2 r \frac{\partial}{\partial r}+3\right)\left(r \frac{\partial}{\partial r}+1\right) \Phi_{3}^{\prime}(x), \\
\Psi_{2}^{\prime}(x) & =\left[\sigma_{1}\left(r \frac{\partial}{\partial r}+3\right)-1\right] \Phi_{2}^{\prime}(x)+\sigma_{2}\left(r \frac{\partial}{\partial r}+3\right) \Phi_{3}^{\prime}(x),
\end{aligned}
$$




$$
\Psi_{2}^{\prime \prime}(x)=\sigma_{3}\left(r \frac{\partial}{\partial r}+3\right) \Phi_{2}^{\prime}(x)+\left[\sigma_{4}\left(r \frac{\partial}{\partial r}+3\right)-1\right] \Phi_{3}^{\prime}(x),
$$

where $\Delta \Phi_{j}^{\prime}(x)=0, j=2,3$.

Substituting these values of the functions $\theta^{\prime}, \theta^{\prime \prime}, \Psi_{2}^{\prime}, \Psi_{3}^{\prime}$ into (3.6) and (3.4), we obtain

$$
\begin{aligned}
u^{\prime}(x)= & \operatorname{grad} \Phi_{1}(x)-x\left(2 r \frac{\partial}{\partial r}+3\right)\left[\left(2 \sigma_{1}-1\right) \Phi_{2}^{\prime}(x)+2 \sigma_{2} \Phi_{3}^{\prime}(x)\right] \\
& +\frac{1}{2} \operatorname{grad} r^{2}\left(r \frac{\partial}{\partial r}+3\right)\left[\left(2 \sigma_{1}-1\right) \Phi_{2}^{\prime}(x)+2 \sigma_{2} \Phi_{3}^{\prime}(x)\right] \\
& +\frac{1}{2} \operatorname{grad} r^{2}\left(r \frac{\partial}{\partial r}+1\right) \Phi_{2}^{\prime}(x)+\operatorname{rot}\left(x \Phi_{5}(x)\right), \\
u^{\prime \prime}(x)= & \operatorname{grad} \Phi_{4}(x)-x\left(2 r \frac{\partial}{\partial r}+3\right)\left[2 \sigma_{3} \Phi_{2}^{\prime}(x)+\left(2 \sigma_{4}-1\right) \Phi_{3}^{\prime}(x)\right] \\
& +\frac{1}{2} \operatorname{grad} r^{2}\left(r \frac{\partial}{\partial r}+3\right)\left[2 \sigma_{3} \Phi_{2}^{\prime}(x)+\left(2 \sigma_{4}-1\right) \Phi_{3}^{\prime}(x)\right] \\
& +\frac{1}{2} \operatorname{grad} r^{2}\left(r \frac{\partial}{\partial r}+1\right) \Phi_{3}^{\prime}(x)+\operatorname{rot}\left(x \Phi_{6}(x)\right) .
\end{aligned}
$$

Let us introduce the notations

$$
\begin{aligned}
& \left(2 \sigma_{1}-1\right) \Phi_{2}^{\prime}(x)+2 \sigma_{2} \Phi_{3}^{\prime}(x)=2 \Phi_{2}(x), \\
& 2 \sigma_{3} \Phi_{2}^{\prime}(x)+\left(2 \sigma_{4}-1\right) \Phi_{3}^{\prime}(x)=2 \Phi_{3}(x) .
\end{aligned}
$$

A solution of this system has the form

$$
\begin{aligned}
& \Phi_{2}^{\prime}(x)=2 \alpha_{1} \Phi_{2}(x)+2 \beta_{1} \Phi_{3}(x), \\
& \Phi_{3}^{\prime}(x)=2 \beta_{2} \Phi_{2}(x)+2 \alpha_{2} \Phi_{3}(x),
\end{aligned}
$$

where

$$
\begin{aligned}
& \alpha_{1}=\frac{1}{d_{1}}\left[c(c+d)-a_{1}\left(a_{2}+b_{2}\right)\right], \quad \beta_{1}=\frac{1}{d_{1}}\left(a_{2} d-c b_{2}\right), \\
& \beta_{2}=\frac{1}{d_{1}}\left(a_{1} d-c b_{1}\right), \quad \alpha_{2}=\left[c(c+d)-a_{2}\left(a_{1}+b_{1}\right)\right],
\end{aligned}
$$

$\Phi_{2}(x), \Phi_{3}(x)$ are scalar harmonic functions.

Substituting the values of $\Phi_{2}^{\prime}(x)$ and $\Phi_{3}^{\prime}(x)$ from (3.9) into (3.8), we obtain

$$
\begin{aligned}
u^{\prime}(x)= & \operatorname{grad} \Phi_{1}(x)+\operatorname{grad} r^{2}\left(r \frac{\partial}{\partial r}+1\right)\left[\alpha_{1} \Phi_{2}+\beta_{1} \Phi_{3}(x)\right] \\
& +\operatorname{rot} \operatorname{rot}\left(x r^{2} \Phi_{2}(x)\right)+\operatorname{rot}\left(x \Phi_{5}(x)\right), \\
u^{\prime \prime}(x)= & \operatorname{grad} \Phi_{4}(x)+\operatorname{grad} r^{2}\left(r \frac{\partial}{\partial r}+1\right)\left[\beta_{2} \Phi_{2}+\alpha_{2} \Phi_{3}(x)\right] \\
& +\operatorname{rot} \operatorname{rot}\left(x r^{2} \Phi_{3}(x)\right)+\operatorname{rot}\left(x \Phi_{6}(x)\right) .
\end{aligned}
$$

The proof of the first parts of the theorem is thereby completed. 
The second part is proved by a direct verification, using the following identities:

$$
\begin{aligned}
& \left(a_{1}+b_{1}\right) \alpha_{1}+(c+d) \beta_{2}=-a_{1}, \\
& \left(a_{1}+b_{1}\right) \beta_{1}+(c+d) \alpha_{2}=-c, \\
& (c+d) \alpha_{1}+\left(a_{2}+b_{2}\right) \beta_{2}=-c, \\
& (c+d) \beta_{1}+\left(a_{2}+b_{2}\right) \alpha_{2}=-a_{2} .
\end{aligned}
$$

This completes the proof of Theorem 3.1.

In proving Theorem 3.1, we have used the following fact [7].

Theorem 3.3. A regular solution of system (1.1) has continuous partial derivatives of any order at an arbitrary point not belonging to $\partial \Omega$.

\section{Solution of Problem $(N)^{+}$}

A solution of this problem will be sought for in form (3.1), where the harmonic functions $\Phi_{j}(x), j=1, \ldots, 6$, are presented in the form [8]

$$
\Phi_{j}(x)=\sum_{k=0}^{\infty} \sum_{m=-k}^{k}\left(\frac{r}{R}\right)^{k} Y_{k}^{(m)}(\vartheta, \varphi) A_{m k}^{(j)}, \quad j=1, \ldots, 6,
$$

where $A_{m k}^{(j)}$ are the constants we seek for, $(r, \vartheta, \varphi)$ are the spherical coordinates of a point $x \in \Omega^{+}$,

$$
Y_{k}^{(m)}(\vartheta, \varphi)=\sqrt{\frac{2 k+1}{4 \pi} \cdot \frac{(k-m) !}{(k+m) !}} P_{k}^{(m)}(\cos \vartheta) e^{i m \varphi},
$$

$P_{k}^{(m)}(\cos \vartheta)$ is the adjoint Legendre function of $k$-th kind and $m$-th order.

We require of the harmonic functions $\Phi_{j}(x), j=1,4,5,6$, to satisfy the condition

$$
\int_{\partial \Omega^{\prime}} \Phi_{j}(x) d s=0, \quad j=1,4,5,6
$$

where $\partial \Omega^{\prime}$ is the sphere with center at the origin and radius $R_{1}\left(0<R_{1}<R\right)$.

Putting the values of the function $\Phi_{j}(x), j=1,4,5,6$, from (4.1) into (4.2) and taking into account the equalities

$$
\int_{\partial \Omega^{\prime}} Y_{k}^{(m)}(\vartheta, \varphi) d s= \begin{cases}2 \sqrt{\pi} R_{1}^{2}, & k=m=0 \\ 0 & \text { for other values of } k \text { and } m\end{cases}
$$

we obtain $A_{00}^{(j)}=0, j=1,4,5,6$. 
The substitution of the values of the function $\Phi_{j}(x), j=1, \ldots, 6$, from (4.1) into (3.1) gives

$$
\begin{gathered}
u^{\prime}(x)=\sum_{k=0}^{\infty} \sum_{m=-k}^{k}\left\{u_{m k}^{(1)}(r) X_{m k}(\vartheta, \varphi)+\sqrt{k(k+1)}\left[v_{m k}^{(1)}(r) Y_{m k}(\vartheta, \varphi)\right.\right. \\
\left.\left.+w_{m k}^{(1)}(r) Z_{m k}(\vartheta, \varphi)\right]\right\} \\
u^{\prime \prime}(x)=\sum_{k=0}^{\infty} \sum_{m=-k}^{k}\left\{u_{m k}^{(2)}(r) X_{m k}(\vartheta, \varphi)+\sqrt{k(k+1)}\left[v_{m k}^{(2)}(r) Y_{m k}(\vartheta, \varphi)\right.\right. \\
\left.\left.+w_{m k}^{(2)}(r) Z_{m k}(\vartheta, \varphi)\right]\right\}
\end{gathered}
$$

where $([2],[6],[9])$

$$
\begin{aligned}
X_{m k}(\vartheta, \varphi) & =e_{r} Y_{k}^{(m)}(\vartheta, \varphi), \quad k \geq 0, \\
Y_{m k}(\vartheta, \varphi) & =\frac{1}{\sqrt{k(k+1)}}\left(e_{\vartheta} \frac{\partial}{\partial \vartheta}+\frac{e_{\varphi}}{\sin \vartheta} \frac{\partial}{\partial \varphi}\right) Y_{k}^{(m)}(\vartheta, \varphi), \quad k \geq 1, \\
Z_{m k}(\vartheta, \varphi) & =\frac{1}{\sqrt{k(k+1)}}\left(\frac{e_{\vartheta}}{\sin \vartheta} \frac{\partial}{\partial \varphi}-e_{\varphi} \frac{\partial}{\partial \vartheta}\right) Y_{k}^{(m)}(\vartheta, \varphi), \quad k \geq 1,
\end{aligned}
$$

$|m| \leq k, e_{r}, e_{\vartheta}, e_{\varphi}$ are the unit orthogonal vectors:

$$
\begin{aligned}
e_{r}= & (\cos \varphi \sin \vartheta, \sin \varphi \sin \vartheta, \cos \vartheta), \\
e_{\vartheta}= & (\cos \varphi \cos \vartheta, \sin \varphi \cos \vartheta,-\sin \vartheta), \\
e_{\varphi}= & (-\sin \varphi, \cos \varphi, 0), \\
u_{m k}^{(j)}(r)= & \frac{k}{R}\left(\frac{r}{R}\right)^{k-1} A_{m k}^{(3 j-2)}+R(k+1)\left[(k+2)\left(\alpha_{j}+1\right)-2\right]\left(\frac{r}{R}\right)^{k+1} A_{m k}^{(j+1)} \\
& +\beta_{j} R(k+1)(k+2)\left(\frac{r}{R}\right)^{k+1} A_{m k}^{(4-j)}, \quad k \geq 0, \\
& +\beta_{j} R(k+1)\left(\frac{r}{R}\right)^{k+1} A_{m k}^{(4-j)}, \quad k \geq 1, \\
v_{m k}^{(j)}(r)= & \frac{1}{R}\left(\frac{r}{R}\right)^{k-1} A_{m k}^{(3 j-2)}+R\left[(k+1)\left(\alpha_{j}+1\right)+2\right]\left(\frac{r}{R}\right)^{k+1} A_{m k}^{(j+1)} \\
w_{m k}^{(j)}(r)= & \left(\frac{r}{R}\right)^{k} A_{m k}^{(j+4)}, \quad k \geq 1, \quad j=1,2 .
\end{aligned}
$$

Since $n(x)=e_{r}$ we obtain

$$
\begin{gathered}
n(x) \cdot X_{m k}(\vartheta, \varphi)=Y_{k}^{(m)}(\vartheta, \varphi), \quad n(x) \cdot Y_{m k}(\vartheta, \varphi)=0 \\
n(x) \cdot Z_{m k}(\vartheta, \varphi)=0 .
\end{gathered}
$$


Taking this into account, from (4.3) we obtain

$$
\begin{aligned}
& n(x) \cdot u^{\prime}(x)=\sum_{k=0}^{\infty} \sum_{m=-k}^{k} u_{m k}^{(1)}(r) Y_{k}^{(m)}(\vartheta, \varphi), \\
& n(x) \cdot u^{\prime \prime}(x)=\sum_{k=0}^{\infty} \sum_{m=-k}^{k} u_{m k}^{(2)}(r) Y_{k}^{(m)}(\vartheta, \varphi) .
\end{aligned}
$$

Applying the operation rot to both sides of each equation of system (4.3), we obtain

$$
\begin{aligned}
& n(x) \times \operatorname{rot} u^{\prime}(x) \\
& =\sum_{k=1}^{\infty} \sum_{m=-k}^{k} \sqrt{k(k+1)}\left[\widetilde{v}_{m k}^{(1)}(r) Y_{m k}(\vartheta, \varphi)+\widetilde{w}_{m k}^{(1)}(r) Z_{m k}(\vartheta, \varphi)\right], \\
& n(x) \times \operatorname{rot} u^{\prime \prime}(x) \\
& =\sum_{k=1}^{\infty} \sum_{m=-k}^{k} \sqrt{k(k+1)}\left[\widetilde{v}_{m k}^{(2)}(r) Y_{m k}(\vartheta, \varphi)+\widetilde{w}_{m k}^{(2)}(r) Z_{m k}(\vartheta, \varphi)\right],
\end{aligned}
$$

where

$$
\begin{aligned}
& \widetilde{v}_{m k}^{(j)}(r)=-2(2 k+3)\left(\frac{r}{R}\right)^{k} A_{m k}^{(j+1)}, \\
& \widetilde{w}_{m k}^{(j)}(r)=-\frac{k+1}{R}\left(\frac{r}{R}\right)^{k-1} A_{m k}^{(j+4)}, \quad k \geq 1, \quad j=1,2 .
\end{aligned}
$$

Since on the sphere $\Sigma_{1}$ the sets $\left\{Y_{k}^{(m)}(\vartheta, \varphi)\right\}_{|m| \leq k, k=\overline{0, \infty}}$ and $\left\{X_{m k}(\vartheta, \varphi)\right.$, $\left.Y_{m k}(\vartheta, \varphi), Z_{m k}(\vartheta, \varphi)\right\}_{|m| \leq k, k=\overline{0, \infty}}$ form a complete orthonormal system in the space $L_{2}$ and using the sufficient condition of smoothness, we can represent the function $f_{4}^{(j}(z)$ and the vector $f^{(j)}(z)$ as Fourier series

$$
\begin{aligned}
f_{4}^{(j)}(z) & =\sum_{k=0}^{\infty} \sum_{m=-k}^{k} \alpha_{m k}^{(j)} Y_{k}^{(m)}(\vartheta, \varphi) \\
f^{(j)}(z) & =\sum_{k=0}^{\infty} \sum_{m=-k}^{k}\left\{\widetilde{\alpha}_{m k}^{(j)} X_{m k}(\vartheta, \varphi)+\sqrt{k(k+1)}\left[\beta_{m k}^{(j)} Y_{m k}(\vartheta, \varphi)\right.\right. \\
& \left.\left.\quad+\gamma_{m k}^{(j)} Z_{m k}(\vartheta, \varphi)\right]\right\}, \quad j=1,2,
\end{aligned}
$$

where $\widetilde{\alpha}_{m k}^{(j)}, \alpha_{m k}^{(j)}, \beta_{m k}^{(j)}, \gamma_{m k}^{(j)}$ are Fourier coefficients. Taking into account that $n(z) \cdot f^{(j)}(z)=0, j=1,2$, from $(4.7)$ we obtain

$$
f^{(j)}(z)=\sum_{k=1}^{\infty} \sum_{m=-k}^{k} \sqrt{k(k+1)}\left[\beta_{m k}^{(j)} Y_{m k}(\vartheta, \varphi)+\gamma_{m k}^{(j)} Z_{m k}(\vartheta, \varphi)\right], \quad j=1,2 .
$$

Passing on both sides of equalities (4.4) and (4.5) to the limit as $x \rightarrow z \in \partial \Omega$ and taking into account the boundary condition of Problem $(N)^{+}$and also 
formulas (4.6) and (4.8), for the unknown constants $A_{m k}^{(j)}, j=1, \ldots, 6$, we obtain the following systems of algebraic equations:

$$
\begin{aligned}
& \alpha_{1} A_{00}^{(2)}+\beta_{1} A_{00}^{(3)}=\frac{1}{2 R} a_{00}^{(1)}, \\
& \beta_{2} A_{00}^{(2)}+\alpha_{2} A_{00}^{(3)}=\frac{1}{2 R} a_{00}^{(2)}, \\
& \frac{k}{R} A_{m k}^{(1)}+R(k+1)\left[(k+2)\left(\alpha_{1}+1\right)-2\right] A_{m k}^{(2)} \\
& \quad+\beta_{1} R(k+1)(k+2) A_{m k}^{(3)}=\alpha_{m k}^{(1)}, \\
& \quad+R(k+1)\left[(k+2)\left(\alpha_{2}+1\right)-2\right] A_{m k}^{(3)}=\alpha_{m k}^{(2)}, \\
& \frac{k}{R} A_{m k}^{(4)}+\beta_{2} R(k+1)(k+2) A_{m k}^{(2)} \\
& 2(2 k+3) A_{m k}^{(2)}=-\beta_{m k}^{(1)}, \\
& 2(2 k+3) A_{m k}^{(3)}=-\beta_{m k}^{(2)}, \\
& \frac{k+1}{R} A_{m k}^{(5)}=-\gamma_{m k}^{(1)}, \\
& \frac{k+1}{R} A_{m k}^{(6)}=-\gamma_{m k}^{(2)}, \quad k \geq 1 .
\end{aligned}
$$

By solving systems (4.9) and (4.10) we have

$$
\begin{aligned}
& A_{00}^{(2)}=\frac{d_{1}}{2 R d_{2}}\left(\alpha_{2} \alpha_{00}^{(1)}-\beta_{1} \alpha_{00}^{(2)}\right), \\
& A_{00}^{(3)}=\frac{d_{1}}{2 R d_{2}}\left(\alpha_{1} \alpha_{00}^{(2)}-\beta_{2} \alpha_{00}^{(1)}\right), \\
& A_{m k}^{(2)}=-\frac{1}{2(2 k+3)} \beta_{m k}^{(1)}, \quad A_{m k}^{(3)}=-\frac{1}{2(2 k+3)} \beta_{m k}^{(2)}, \\
& A_{m k}^{(5)}=-\frac{R}{k+1} \gamma_{m k}^{(1)}, \quad A_{m k}^{(6)}=-\frac{R}{k+1} \gamma_{m k}^{(2)}, \\
& A_{m k}^{(1)}=\frac{R}{k} \alpha_{m k}^{(1)}+\frac{(k+1) R^{2}}{2 k(2 k+3)}\left[\left((k+2)\left(\alpha_{1}+1\right)-2\right) \beta_{m k}^{(1)}+\beta_{1}(k+2) \beta_{m k}^{(2)}\right], \\
& A_{m k}^{(4)}=\frac{R}{k} \alpha_{m k}^{(2)}+\frac{(k+1) R^{2}}{2 k(2 k+3)}\left[\beta_{2}(k+2) \beta_{m k}^{(1)}+\left((k+2)\left(\alpha_{2}+1\right)-2\right) \beta_{m k}^{(2)}\right] .
\end{aligned}
$$

The substitution of these values of the constants $A_{m k}^{(j)}, j=1, \ldots, 6$, into (4.3), (4.4) and (4.5) gives

$$
\begin{aligned}
u^{\prime}(x)= & \sum_{k=0}^{\infty} \sum_{m=-k}^{k}\left(\frac{r}{R}\right)^{k+1}\left\{\alpha_{m k}^{(1)} X_{m k}(\vartheta, \varphi)+\sqrt{k(k+1)}\left[\frac{1}{k} \alpha_{m k}^{(1)}\right.\right. \\
& \left.\left.+\frac{R}{k(2 k+3)}\left((k+1) \alpha_{1}-k\right) \beta_{m k}^{(1)}+\beta_{1}(k+1) \beta_{m k}^{(2)}\right)\right] Y_{m k}(\vartheta, \varphi)
\end{aligned}
$$




$$
\begin{aligned}
& \left.-\sqrt{k(k+1)} \frac{R^{2}}{r(k+1)} \gamma_{m k}^{(1)} Z_{m k}(\vartheta, \varphi)\right\} \\
& +\frac{R^{2}-r^{2}}{R^{3}} \sum_{k=1}^{\infty} \sum_{m=-k}^{k}\left(\frac{r}{R}\right)^{k-1}\left[k X_{m k}(\vartheta, \varphi)\right. \\
& \left.+\sqrt{k(k+1)} Y_{m k}(\vartheta, \varphi)\right] A_{m k}^{(1)} \\
& u^{\prime \prime}(x)=\sum_{k=0}^{\infty} \sum_{m=-k}^{k}\left(\frac{r}{R}\right)^{k+1}\left\{\alpha_{m k}^{(2)} X_{m k}(\vartheta, \varphi)+\sqrt{k(k+1)}\left[\frac{1}{k} \alpha_{m k}^{(2)}\right.\right. \\
& \left.+\frac{R}{k(2 k+3)}\left(\beta_{2}(k+1) \beta_{m k}^{(1)}+\left((k+1) \alpha_{2}-k\right) \beta_{m k}^{(2)}\right)\right] Y_{m k}(\vartheta, \varphi) \\
& \left.-\sqrt{k(k+1)} \frac{R^{2}}{r(k+1)} \gamma_{m k}^{(2)} Z_{m k}(\vartheta, \varphi)\right\} \\
& +\frac{R^{2}-r^{2}}{R^{3}} \sum_{k=1}^{\infty} \sum_{m=-k}^{k}\left(\frac{r}{R}\right)^{k-1}\left[k X_{m k}(\vartheta, \varphi)\right. \\
& \left.+\sqrt{k(k+1)} Y_{m k}(\vartheta, \varphi)\right] A_{m k}^{(4)} \\
& n(x) \cdot u^{\prime}(x)=\sum_{k=0}^{\infty} \sum_{m=-k}^{k}\left(\frac{r}{R}\right)^{k+1} \alpha_{m k}^{(1)} Y_{k}^{(m)}(\vartheta, \varphi) \\
& +\frac{R^{2}-r^{2}}{R^{3}} \sum_{k=1}^{\infty} \sum_{m=-k}^{k} k\left(\frac{r}{R}\right)^{k-1} Y_{k}^{(m)}(\vartheta, \varphi) A_{m k}^{(1)}, \\
& n(x) \cdot u^{\prime \prime}(x)=\sum_{k=0}^{\infty} \sum_{m=-k}^{k}\left(\frac{r}{R}\right)^{k+1} \alpha_{m k}^{(2)} Y_{k}^{(m)}(\vartheta, \varphi) \\
& +\frac{R^{2}-r^{2}}{R^{3}} \sum_{k=1}^{\infty} \sum_{m=-k}^{k} k\left(\frac{r}{R}\right)^{k-1} Y_{k}^{(m)}(\vartheta, \varphi) A_{m k}^{(4)},
\end{aligned}
$$

$$
\begin{aligned}
& n(x) \times \operatorname{rot} u^{\prime}(x) \\
& =\sum_{k=1}^{\infty} \sum_{m=-k}^{k} \sqrt{k(k+1)}\left(\frac{r}{R}\right)^{k}\left[\beta_{m k}^{(1)} Y_{m k}(\vartheta, \varphi)+\frac{R}{r} \gamma_{m k}^{(1)} Z_{m k}(\vartheta, \varphi)\right], \\
& n(x) \times \operatorname{rot} u^{\prime \prime}(x) \\
& =\sum_{k=1}^{\infty} \sum_{m=-k}^{k} \sqrt{k(k+1)}\left(\frac{r}{R}\right)^{k}\left[\beta_{m k}^{(2)} Y_{m k}(\vartheta, \varphi)+\frac{R}{r} \gamma_{m k}^{(2)} Z_{m k}(\vartheta, \varphi)\right] .
\end{aligned}
$$

The following statement is true [2] 
Theorem 4.1. The vectors $X_{m k}(\vartheta, \varphi), Y_{m k}(\vartheta, \varphi), Z_{m k}(\vartheta, \varphi)$ admit the following estimates for any $k \geq 0$ :

$$
\begin{aligned}
& \left|X_{m k}(\vartheta, \varphi)\right| \leq \sqrt{\frac{2 k+1}{4 \pi}}, \quad k \geq 0, \\
& \left|Y_{m k}(\vartheta, \varphi)\right|<\sqrt{\frac{2 k(k+1)}{2 k+1}}, \quad k \geq 1, \\
& \left|Z_{m k}(\vartheta, \varphi)\right|<\sqrt{\frac{2 k(k+1)}{2 k+1}}, \quad k \geq 1 .
\end{aligned}
$$

Moreover, as is known [8],

$$
\left|Y_{k}^{(m)}(\vartheta, \varphi)\right| \leq \sqrt{\frac{2 k+1}{4 \pi}}, \quad k \geq 0
$$

By virtue of these estimates, series (4.11)-(4.13) will be absolutely and uniformly convergent in the domain $\bar{\Omega}^{+}$if we prove the convergence of the majorizing series

$$
\alpha \sum_{k=k_{0}}^{\infty} k^{\frac{3}{2}}\left[\left|\alpha_{m k}^{(j)}\right|+k\left(\left|\beta_{m k}^{(1)}\right|+\left|\beta_{m k}^{(2)}\right|\left|\gamma_{m k}^{(j)}\right|\right)\right], \quad j=1,2,
$$

where $\alpha$ is a positive constant not depending on $k$.

Series (4.16) converges if we require of the coefficients $\alpha_{m k}^{(j)}, \beta_{m k}^{(j)}, \gamma_{m k}^{(m)}, j=1,2$, to have order

$$
\alpha_{m k}^{(j)}=O\left(k^{-3}\right), \quad \beta_{m k}^{(j)}=O\left(k^{-4}\right), \quad \gamma_{m k}^{(j)}=O\left(k^{-4}\right), \quad j=1,2 .
$$

The following theorem is true [2].

Theorem 4.2. If $f^{(j)}(z) \in C^{l}(\partial \Omega), j=1,2$, then the coefficients $\widetilde{\alpha}_{m k}^{(j)}$, $\beta_{m k}^{(j)}$, $\gamma_{m k}^{(j)}$ admit the following estimates:

$$
\widetilde{\alpha}_{m k}^{(j)}=O\left(k^{-l}\right), \quad \beta_{m k}^{(j)}=O\left(k^{-l-1}\right), \quad \gamma_{m k}^{(j)}=O\left(k^{-l-1}\right), \quad l \geq 1 .
$$

Analogously, if $f_{4}^{(j)}(z) \in C^{l}(\partial \Omega), j=1,2$, then the estimate

$$
\alpha_{m k}^{(j)}=O\left(k^{-l}\right), \quad j=1,2,
$$

is valid [5].

With these properties of Fourier coefficients taken into account, we conclude that the coefficients $\alpha_{m k}^{(j)}, \beta_{m k}^{(j)}, \gamma_{m k}^{(j)}, j=1,2$, admit estimates (4.17) if the boundary vector functions satisfy the following sufficient conditions:

$$
f_{4}^{(j)}(z) \in C^{3}(\partial \Omega), \quad f^{(j)}(z) \in C^{3}(\partial \Omega), \quad j=1,2 .
$$

Passing on both sides of equalities (4.12) and (4.13) to the limit as $x \rightarrow z \in$ $\partial \Omega(r \rightarrow R)$, we obtain

$$
\left[n(z) \cdot u^{\prime}(z)\right]^{+}=\sum_{k=0}^{\infty} \sum_{m=-k}^{k} \alpha_{m k}^{(1)} Y_{k}^{(m)}(\vartheta, \varphi)=f_{4}^{(1)}(z)
$$




$$
\begin{aligned}
& {\left[n(z) \cdot u^{\prime \prime}(z)\right]^{+}=\sum_{k=0}^{\infty} \sum_{m=-k}^{k} \alpha_{m k}^{(2)} Y_{k}^{(m)}(\vartheta, \varphi)=f_{4}^{(2)}(z)} \\
& {\left[n(z) \times \operatorname{rot} u^{\prime}(z)\right]^{+}} \\
& \quad=\sum_{k=1}^{\infty} \sum_{m=-k}^{k} \sqrt{k(k+1)}\left[\beta_{m k}^{(1)} Y_{k}^{(m)}(\vartheta, \varphi)+\gamma_{m k}^{(1)} Z_{m k}(\vartheta, \varphi)\right]=f^{(1)}(z), \\
& {\left[n(z) \times \operatorname{rot} u^{\prime \prime}(z)\right]^{+}} \\
& \quad=\sum_{k=1}^{\infty} \sum_{m=-k}^{k} \sqrt{k(k+1)}\left[\beta_{m k}^{(2)} Y_{k}^{(m)}(\vartheta, \varphi)+\gamma_{m k}^{(2)} Z_{m k}(\vartheta, \varphi)\right]=f^{(2)}(z)
\end{aligned}
$$

Thus, if $f_{4}^{(j)}(z) \in C^{3}(\partial \Omega), f^{(j)}(z) \in C^{3}(\partial \Omega), j=1,2$, then the vector $U=$ $\left(u^{\prime}, u^{\prime \prime}\right)$ represented by $(4.11)$ is a regular solution of Problem $(N)^{+}$.

\section{Solution of Problem $(N)^{-}$}

A solution of this problem will be sought for in form (3.1), where the harmonic functions $\Phi_{j}(x), j=1, \ldots, 6$, are presented in the form [8]

$$
\Phi_{j}(x)=\sum_{k=0}^{\infty} \sum_{m=-k}^{k}\left(\frac{R}{r}\right)^{k+1} Y_{k}^{(m)}(\vartheta, \varphi) A_{m k}^{(j)}, \quad j=1, \ldots, 6,
$$

where $A_{m k}^{(j)}$ are the constants we seek for.

We assume that these harmonic functions satisfy the conditions

$$
\int_{\partial \Omega^{\prime}} \Phi_{j}(z) d s=0, \quad j=2,3,5,6
$$

where $\partial \Omega^{\prime}$ is the sphere with center at the origin and radius $R_{1}\left(R<R_{1}<+\infty\right)$.

Putting the values of the function $\Phi_{j}(x), j=2,3,5,6$, from (5.1) into (5.2), we obtain $A_{00}^{(j)}=0, j=2,3,5,6$.

The substitution of the values of the function $\Phi_{j}(x), j=1, \ldots, 6$, from (5.1) into (3.1) enables us to represent the vectors $u^{\prime}(x)$ and $u^{\prime \prime}(x)$ as series (4.3), where

$$
\begin{aligned}
u_{m k}^{(j)}(r)= & -\frac{k+1}{R}\left(\frac{R}{r}\right)^{k+2} A_{m k}^{(3 j-2)} \\
& +R k\left[(k-1)\left(\alpha_{j}+1\right)+2\right]\left(\frac{R}{r}\right)^{k} A_{m k}^{(j+1)} \\
& +\beta_{j} k(k-1) R\left(\frac{R}{r}\right)^{k} A_{m k}^{(4-j)}, \quad k \geq 0, \\
v_{m k}^{(j)}(r)= & \frac{1}{R}\left(\frac{R}{r}\right)^{k+2} A_{m k}^{(3 j-2)}-R\left[k\left(\alpha_{j}+1\right)-2\right]\left(\frac{R}{r}\right)^{k} A_{m k}^{(j+1)}
\end{aligned}
$$




$$
\begin{gathered}
-\beta_{j} k R\left(\frac{R}{r}\right)^{k} A_{m k}^{(4-j)}, \quad k \geq 1, \\
w_{m k}^{(j)}(r)=\left(\frac{R}{r}\right)^{k+1} A_{m k}^{(j+4)}, \quad k \geq 1, \quad j=1,2 .
\end{gathered}
$$

Calculating $n(x) \cdot u^{\prime}(x), n(x) \cdot u^{\prime \prime}(x), n(x) \times \operatorname{rot} u^{\prime}(x), n(x) \times \operatorname{rot} u^{\prime \prime}(x)$ with the aid of (4.3) and (5.3), we obtain equalities (4.4) and (4.5), where $u_{m k}^{(j)}(r)$, $j=1,2$, has form (5.3), and

$$
\begin{aligned}
\widetilde{v}_{m k}^{(j)}(r) & =2(2 k-1)\left(\frac{R}{r}\right)^{k+1} A_{m k}^{(j+1)} \\
\widetilde{w}_{m k}^{(j)}(r) & =\frac{k}{R}\left(\frac{R}{r}\right)^{k+2} A_{m k}^{(j+4)}, \quad k \geq 1, \quad j=1,2 .
\end{aligned}
$$

By the boundary conditions of problem $(N)^{-}$, for the constants $A_{m k}^{(j)}, j=$ $1, \ldots, 6$, we obtain the following system of algebraic equations:

$$
\begin{aligned}
& A_{00}^{(1)}=-R \alpha_{00}^{(1)}, \quad A_{00}^{(4)}=-R \alpha_{00}^{(2)}, \\
& -\frac{k+1}{R} A_{m k}^{(1)}+R k\left[(k-1)\left(\alpha_{1}+1\right)+2\right] A_{m k}^{(2)}+\beta_{1} k(k-1) R A_{m k}^{(3)}=\alpha_{m k}^{(1)}, \\
& -\frac{k+1}{R} A_{m k}^{(4)}+\beta_{2} k(k-1) R A_{m k}^{(2)}+R k\left[(k-1)\left(\alpha_{2}+1\right)+2\right] A_{m k}^{(3)}=\alpha_{m k}^{(2)}, \\
& 2(2 k-1) A_{m k}^{(2)}=\beta_{m k}^{(1)}, \quad 2(2 k-1) A_{m k}^{(3)}=\beta_{m k}^{(2)}, \\
& \frac{k}{R} A_{m k}^{(5)}=\gamma_{m k}^{(1)}, \quad \frac{k}{R} A_{m k}^{(6)}=\gamma_{m k}^{(2)}, \quad k \geq 1 .
\end{aligned}
$$

A solution of this system has the form

$$
\begin{aligned}
A_{m k}^{(2)}= & \frac{1}{2(2 k-1)} \beta_{m k}^{(1)}, \quad A_{m k}^{(3)}=\frac{1}{2(2 k-1)} \beta_{m k}^{(2)}, \\
A_{m k}^{(5)}= & \frac{R}{k} \gamma_{m k}^{(1)}, \quad A_{m k}^{(6)}=\frac{R}{k} \gamma_{m k}^{(2)}, \\
A_{m k}^{(1)}= & -\frac{R}{k+1} \alpha_{m k}^{(1)} \\
& +\frac{R^{2} k}{2(k+1)(2 k-1)}\left[\left((k-1)\left(\alpha_{1}+1\right)+2\right) \beta_{m k}^{(1)}+\beta_{1}(k-1) \beta_{m k}^{(2)}\right], \\
A_{m k}^{(4)}= & -\frac{R}{k+1} \alpha_{m k}^{(2)} \\
& +\frac{R^{2} k}{2(k+1)(2 k-1)}\left[\beta_{2}(k-1) \beta_{m k}^{(1)}+\left((k-1)\left(\alpha_{2}+1\right)+2\right) \beta_{m k}^{(2)}\right] .
\end{aligned}
$$


The substitution of the values of the constant $A_{m k}^{(j)}, j=1, \ldots, 6$, from (5.5) into (5.3), (5.4), (4.3)-(4.5) gives

$$
\begin{aligned}
& u^{\prime}(x)=\sum_{k=0}^{\infty} \sum_{m=-k}^{k}\left(\frac{R}{r}\right)^{k}\left\{\alpha_{m k}^{(1)} X_{m k}(\vartheta, \varphi)-\sqrt{k(k+1)}\left[\frac{1}{k+1} \alpha_{m k}^{(1)}\right.\right. \\
& \left.\left.+\frac{R\left(k\left(\alpha_{1}-1\right)-1\right)}{(k+1)(2 k-1)} \beta_{m k}^{(1)}+\frac{R k \beta_{1}}{(k+1)(2 k-1)} \beta_{m k}^{(2)}\right)\right] Y_{m k}(\vartheta, \varphi) \\
& \left.+\sqrt{k(k+1)} \frac{R^{2}}{k r} \gamma_{m k}^{(1)} Z_{m k}(\vartheta, \varphi)\right\}+\frac{R^{2}-r^{2}}{R^{3}} \sum_{k=0}^{\infty} \sum_{m=-k}^{k}\left(\frac{R}{r}\right)^{k+2} \\
& \times\left[-(k+1) X_{m k}(\vartheta, \varphi)+\sqrt{k(k+1)} Y_{m k}(\vartheta, \varphi)\right] A_{m k}^{(1)}, \\
& u^{\prime \prime}(x)=\sum_{k=0}^{\infty} \sum_{m=-k}^{k}\left(\frac{R}{r}\right)^{k}\left\{\alpha_{m k}^{(2)} X_{m k}(\vartheta, \varphi)-\sqrt{k(k+1)}\left[\frac{1}{k+1} \alpha_{m k}^{(2)}\right.\right. \\
& \left.\left.+\frac{R k \beta_{2}}{(k+1)(2 k-1)} \beta_{m k}^{(1)}+\frac{R\left(k\left(\alpha_{2}-1\right)-1\right)}{(k+1)(2 k-1)} \beta_{m k}^{(2)}\right)\right] Y_{m k}(\vartheta, \varphi) \\
& \left.+\sqrt{k(k+1)} \frac{R^{2}}{k r} \gamma_{m k}^{(2)} Z_{m k}(\vartheta, \varphi)\right\}+\frac{R^{2}-r^{2}}{R^{3}} \sum_{k=0}^{\infty} \sum_{m=-k}^{k}\left(\frac{R}{r}\right)^{k+2} \\
& \times\left[-(k+1) X_{m k}(\vartheta, \varphi)+\sqrt{k(k+1)} Y_{m k}(\vartheta, \varphi)\right] A_{m k}^{(4)} \text {; } \\
& n(x) \cdot u^{\prime}(x)=\sum_{k=0}^{\infty} \sum_{m=-k}^{k}\left(\frac{R}{r}\right)^{k} \alpha_{m k}^{(1)} Y_{k}^{(m)}(\vartheta, \varphi) \\
& -\frac{R^{2}-r^{2}}{R^{3}} \sum_{k=0}^{\infty} \sum_{m=-k}^{k}(k+1)\left(\frac{R}{r}\right)^{k+2} Y_{k}^{(m)}(\vartheta, \varphi) A_{m k}^{(1)}, \\
& n(x) \cdot u^{\prime \prime}(x)=\sum_{k=0}^{\infty} \sum_{m=-k}^{k}\left(\frac{R}{r}\right)^{k} \alpha_{m k}^{(2)} Y_{k}^{(m)}(\vartheta, \varphi) \\
& -\frac{R^{2}-r^{2}}{R^{3}} \sum_{k=0}^{\infty} \sum_{m=-k}^{k}(k+1)\left(\frac{R}{r}\right)^{k+2} Y_{k}^{(m)}(\vartheta, \varphi) A_{m k}^{(4)} \text {; }
\end{aligned}
$$

$$
\begin{aligned}
& n(x) \times \operatorname{rot} u^{\prime}(x) \\
& =\sum_{k=1}^{\infty} \sum_{m=-k}^{k} \sqrt{k(k+1)}\left(\frac{R}{r}\right)^{k+1}\left[\beta_{m k}^{(1)} Y_{m k}(\vartheta, \varphi)+\frac{R}{r} \gamma_{m k}^{(1)} Z_{m k}(\vartheta, \varphi)\right], \\
& n(x) \times \operatorname{rot} u^{\prime \prime}(x) \\
& \quad=\sum_{k=1}^{\infty} \sum_{m=-k}^{k} \sqrt{k(k+1)}\left(\frac{R}{r}\right)^{k+1}\left[\beta_{m k}^{(2)} Y_{m k}(\vartheta, \varphi)+\frac{R}{r} \gamma_{m k}^{(2)} Z_{m k}(\vartheta, \varphi)\right] .
\end{aligned}
$$


Series (5.6)-(5.8) are absolutely and uniformly convergent when $f_{4}^{(j)}(z) \in$ $C^{3}(\partial \Omega), f^{(j)}(z) \in C^{3}(\partial \Omega), j=1,2$.

Passing on both sides of equalities (5.7) and (5.8) to the limit as $x \rightarrow z \in \partial \Omega$ and taking into account formulas (4.6) and (4.8), we obtain

$$
\begin{array}{ll}
{\left[n(z) \cdot u^{\prime}(z)\right]^{-}=f_{4}^{(1)}(z),} & {\left[n(z) \cdot u^{\prime \prime}(z)\right]^{-}=f_{4}^{(2)}(z),} \\
{\left[n(z) \times \operatorname{rot} u^{\prime}(z)\right]^{-}=f^{(1)}(z),} & {\left[n(z) \times \operatorname{rot} u^{\prime \prime}(z)\right]^{-}=f^{(2)}(z), \quad x \in \partial \Omega .}
\end{array}
$$

As $r \rightarrow \infty$, formulas (4.3) and (5.3) imply

$$
\begin{aligned}
& u^{\prime}(x)=O\left(r^{-1}\right), \quad u^{\prime \prime}(x)=O\left(r^{-1}\right), \\
& \frac{\partial u^{\prime}(x)}{\partial x_{k}}=O\left(r^{-2}\right), \quad \frac{\partial u^{\prime \prime}(x)}{\partial x_{k}}=O\left(r^{-2}\right), \quad k=1,2,3 .
\end{aligned}
$$

Thus if $f_{4}^{(j)}(z) \in C^{3}(\partial \Omega), f^{(j)}(z) \in C^{3}(\partial \Omega)$, then the vector $U=\left(u^{\prime}, u^{\prime \prime}\right)$ represented by (5.6) is a solution of problem $\left(N^{-}\right)$.

\section{REFERENCES}

1. T. V. Buchukuri and T. G. Gegelia, Boundary value problems in elasticity theory with concentrated singularities. (Russian) Differentsial'nye Uravneniya 25(1989), No. 10, 1746-1755; English transl.: Differential Equations 25(1989), No. 10, 1226-1234 (1990).

2. L. G. Giorgashvili, Solution of the basic boundary value problems of stationary thermoelastic oscillations for domains bounded by spherical surfaces. Georgian Math. J. 4(1997), No. 5, 421-438.

3. L. G. Giorgashvili, Solution of the basic boundary value problems of statics of the moment theory for a ball. (Russian) Trudy Tbiliss. Univ., 1977, No. 185, 91-101.

4. A. E. Green and T. R. Steel, Constitutive equations for interacting continua. Int. J. Eng. Sci. 4(1966), No. 4, 483-500.

5. S. G. Mikhlin, Higher-dimensional singular integrals and integral equations. (Russian) Gosudarstv. Izdat. Fiz.-Mat. Lit., Moscow, 1962.

6. F. M. Mors and G. Feshbah, Methods of theoretical physics, v. 2. (Traslated into Russian) Izdat. Inostr, Literaturi, Moscow, 1960.

7. D. G. Natroshvili, A. J. Dzhagmaidze, and M. G. Svanadze, Some problems in the linear theory of elastic mixtures. (Russian) Tbilis. Gos. Univ., Tbilisi, 1986.

8. A. N. Tikhonov and A. A. SAmarskǐ̆, Equations of mathematical physics. Nauka, Moscow, 1966.

9. A. F. Ulitko, The method of eigenvector functions in three-dimensional problems of the theory of elasticity. Naukova Dumka, Kiev, 1979.

10. L. P. Khoroshun and N. S. Soltanov, Thermoelasticity of two-component mixtures. (Russian) Naukova Dumka, Kiev, 1984.

(Received 8.10.2004)

Authors' address:

Higher Mathematics Chair \# 99

Georgian Technical University

77, M. Kostava St., Tbilisi 0175, Georgia

E-mail: lgiorgashvili@yahoo.com 